\title{
Grid Inertial Response-Based Probabilistic Determination of Energy Storage System Capacity under High Solar Penetration
}

\author{
Meng Yue and Xiaoyu Wang
}

\author{
Sustainable Energy Technologies Department, Brookhaven National Laboratory, Upton, NY 11973-5000, \\ U.S.A.
}

\begin{abstract}
It is well-known that responsive battery energy storage systems (BESSs) are an effective means to improve the grid inertial response to various disturbances including the variability of the renewable generation. One of the major issues associated with its implementation is the difficulty in determining the required BESS capacity mainly due to the large amount of inherent uncertainties that cannot be accounted for deterministically. In this study, a probabilistic approach is proposed to properly size the BESS from the perspective of the system inertial response, as an application of probabilistic risk assessment (PRA). The proposed approach enables a riskinformed decision-making process regarding (1) the acceptable level of solar penetration in a given system and (2) the desired BESS capacity (and minimum cost) to achieve an acceptable grid inertial response with a certain confidence level.

Index Terms - Inertial response, probabilistic risk assessment, solar generation, fast cloud transient, battery energy storage system.
\end{abstract}

\section{INTRODUCTION}

Traditional generating sources and the associated inertia are being displaced by renewable sources that have low or virtually no inertia. The rapidly increasing penetration of the renewables causes a rising concern with the performance of grid inertial response [1], especially under light load conditions. This is because, in addition to the inertia reduction and outages of existing components, large power mismatches caused by the fluctuations of renewable generation may often occur. Moreover, many generators no longer participate in governor responses and the inertial response has been deteriorating constantly in some areas of the U.S. [1].

The degraded inertial response may increase the risk of triggering under-frequency load shedding and cascaded grid outages [1, 2]. An effective means to improve the inertial response is to use energy storage systems (ESSs) because their response speeds are superior to conventional generators. The major difficulty is to determine the needed capacity of the expensive ESSs for reducing the risk associated with the degraded inertial response.

Deterministically, an ESS capacity can be estimated as the product of the system daily (or hourly) load and the number of autonomous days (or hours) [3]. It has been recognized, however, that probabilistic approaches are more effective than the deterministic ones to capture the inherent uncertainties. A number of ESS sizing studies using probabilistic methods can be found in various references, e.g., [3 - 10]. All of these studies either focus on generation planning considering long- term variability [3 - 7] or smooth-out of short-term intermittency of renewables [8 - 9]. A well-being concept was developed in [3] to incorporate deterministic criteria into the reliability evaluation of generation systems considering different ESS capacities. A standalone system was used to investigate the needed capacities of wind turbines, hydrogen storage tank, and electrolyzer considering wind turbine power curve and wind speed distribution in [4]. A statistical unit commitment problem was formulated and solved for optimally sizing battery energy storage system (BESS) for an isolated system in [5]. A risk measure that may be accepted by the power producers was used to determine the ESS capacity based on a number of scenario forecasts [6]. Both uncertainties of renewable generation and hardware failures were considered in a generation planning with and without BESS in [7]. Studies in [8,9] attempt to smooth out the individual renewable generation. Optimal sizing of a hybrid battery and super-capacitor storage system was investigated to smooth out the wind generation in [8]. In [9], the ESS was sized as a function of residue forecasting uncertainty of wind generation.

These studies attempt to address variability of renewable generation without evaluating their impacts in term of the overall system responses to such variability. In [10], sizing BESS was studied based on the monthly frequency variation of a microgrid in the presence of variable wind generation. A sensitivity analysis was performed to numerically achieve the minimum capacity using monthly wind generation data. It should be noted that ref. [11], although irrelevant to the ESS sizing or renewable integration, presented a probabilistic transient stability assessment that is relevant to the inertial response study in this paper.

In this study, a probabilistic approach for sizing BESSs is proposed from the perspective of the transient responses to various disturbances of the entire system. The approach consists of (1) development of probabilistic models for the disturbances related to component failures and sudden drop in solar generation due to fast cloud transients; (2) performance of a Monte Carlo analysis to evaluate grid inertial response using the samples for all credible disturbances of concern under a certain solar penetration level; and (3) calculation of measure(s) defined in terms of grid inertial response (e.g., the frequency nadir or deviation) for different BESS capacities until the inertial response satisfies a pre-defined criterion with an acceptable probability conditioning on the disturbance 
occurrences.

The proposed approach is an application of probabilistic risk assessment (PRA), which is widely used in the nuclear power industry [12]. The major feature of this proposed approach is that it captures uncertainties of major disturbances in the BESS sizing based on the explicit evaluation of the system frequency responses to such disturbances. It should be pointed out that the proposed approach quantifies the probabilistic measure for the impacts on the grid inertial responses. To determine the risk measure, the consequence of the disturbances is not defined here, but may be defined as the potential cost to utilities associated with large frequency deviation. Additional cost-benefit analysis can be done after this study to determine the minimum cost among alternative options for achieving acceptable risk level associated with the inertial responses. It should also be stressed that the objective of this study is to describe a systematic and implantable approach for sizing the ESSs. The numerical results are only for an illustration purpose.

This paper is organized as follows: Section II presents probabilistic models for various disturbances affecting the inertial responses. Failure data collection, development of credible disturbances and the probability calculation are discussed in Section III. The random sample generation scheme and the detailed procedures for BESS capacity determination are shown in Section IV. A case study is performed and the results are presented in Section $\mathrm{V}$ using an example system. Conclusions and future work are described in Section VI.

\section{Probabilistic Modeling of Disturbances AfFecting INERTIAL RESPONSES}

\section{A. A Probabilistic Failure Model for Generator Trips}

The major disturbance affecting the inertia response is a loss of generation or a generator failure. A generator may have different failure modes such as a generator trip and de-rated generation. In this study, only generator trips are modeled. The uncertainties related to a generator trip mainly include its occurrence frequency and the way a generator trips, which can be described by a generator trip model in Fig. 1 .

In Fig. 1, a random variable (r.v) is used to represent the unknown time it takes for the generation to ramp-down from the original value to zero including the sudden trip scenario of a generator. This generator trip model can be implemented by gradually increasing the generator internal impedance until the generator output drops to near zero within the selected time period.

\section{B. A Probabilistic Model for Solar Transients}

Although it is generally believed that a sudden generation change of a large wind farm is very rare (but still possible), a fast cloud-transient induced output variation of a utility scale solar PV plant is likely to occur [1], which is of primary interest for the inertial response studies.

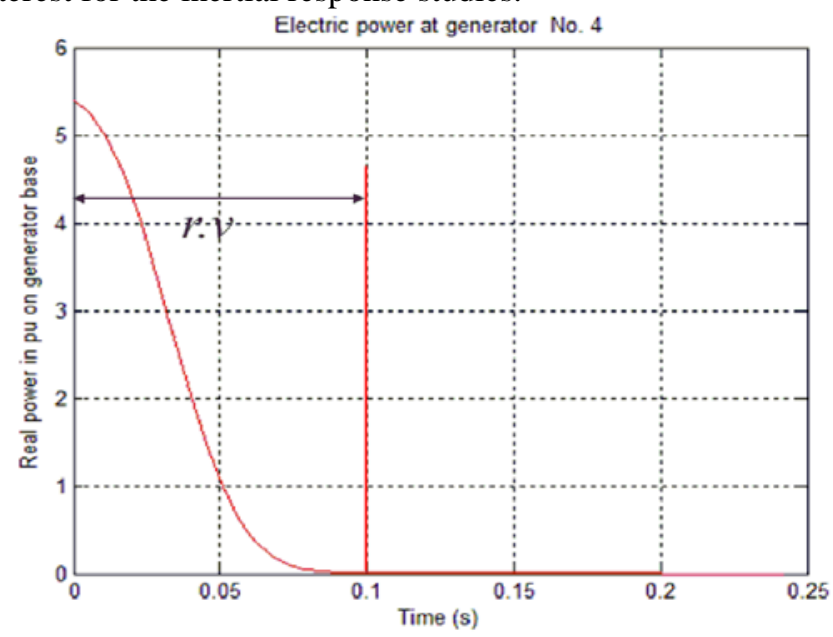

Fig. 1. An example power output curve of a generator trip.

Analyzing the data of actual power output from solar plants is straightforward to capture the statistics of the solar variability. Unfortunately, such data, especially the highresolution measurement data, is expensive and generally unavailable. On the other hand, the solar resource data including solar irradiance and temperature is much easier and cheaper to acquire. Therefore, in this study, it is proposed to analyze the solar irradiance variations caused by fast cloud transients, and feed such data and the electrical circuit model of solar plants to estimate the solar power output statistically [13]. Also, the generic solar model can provide flexibility to evaluate the regional performance of the solar power plants consisting of various solar panels using the weather data.

An example irradiance change pattern induced by a cloud transient is shown in Fig. 2, where the irradiance level drops from its initial value to the lowest value within about 6 seconds. The example fast transient is from actual data collected from a solar farm in the northeast region of the country and, though such big transients are not that often, indicates that cloud transients can be very quick.

To capture the statistics of the cloud transients, a set of random variables may be used to characterize the irradiance variation. The randomness of a cloud transient accounted for in this study includes (1) how often such transients occur; (2) the initial irradiance level $\left(r . v_{1}\right)$; (3) how fast $\left(r . v_{2}\right)$ and how much the irradiance changes over the transient $\left(r . v_{3}\right)$ (or percentage of irradiance changes, $r . v_{4}$ ); and (4) the potential correlations between the different types of randomness. The correlation of the randomness needs to be investigated using actual data, as will be shown in Section III.

Other types of randomness that may need to be modeled include (1) how the irradiance level changes after it reaches the lowest point (e.g., the irradiance level may become flat) and (2) how soon it recovers to the original value. The trip of a solar plant can also modeled similarly by changing the 
irradiance level for all arrays in the plant to zero, i.e., the power injected into the grid decreases to zero within the time frame of a generator trip.

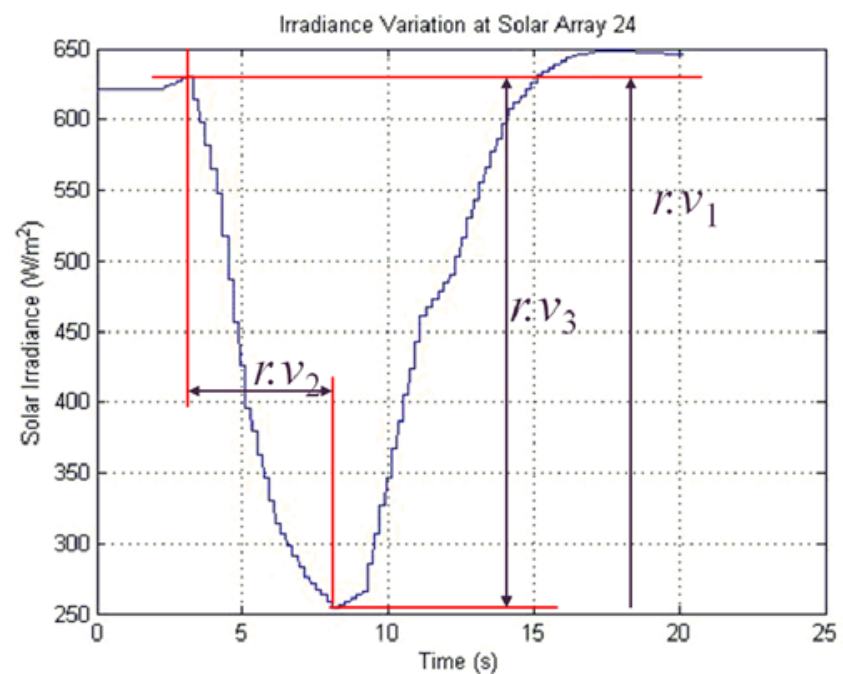

Fig. 2. An example irradiance variation during a fast cloud transient.

Obviously, the solar power generation is proportional to the irradiance level and therefore, this model for solar transients may be applicable to a relatively small solar plant. Since a large solar plant typically spreads over a wide area, a cloud pass-over should only affect the irradiance at the portion of the plant being covered by the cloud. To partially address this issue ${ }^{1}$, a solar plant is modeled in a more detailed manner, i.e., a number of arrays, as will be discussed in Section III.B.

\section{Probabilistic Failure and Transient Models of Other Components}

One important failure is the common cause failure (CCF), i.e., simultaneous failures of multiple components due to, e.g., the same environmental operating condition of two components. This is different from a so-called "dependent failure", which means that a component failure is caused by another one, e.g., the trip of an overloaded transmission line due to the outage of the other parallel transmission line. In this study, the CCFs of two generators or two solar plants are explicitly considered. Dependent failures for generators are very unlikely, and therefore, are not included.

Some other component outages such as transmission lines may also affect the frequency responses. Since the focus of this study is on the inertial response, the probabilistic model for transmission line outages is not considered but a model similar to the one in [11] can be used as needed.

\section{COLLECTION AND ANALYSIS OF DistURBANCE DATA}

The sample generation for a Monte Carlo simulation

\footnotetext{
${ }^{1}$ If the high-resolution solar power variation data is available, the similar probabilistic model is directly applicable. Alternatively, a higher-dimensional model can be developed to also capture the locational irradiance variations.
}

consists of two steps. The first step is to select a single disturbance or a combinational disturbance from a preidentified disturbance list. A combinational disturbance means the simultaneous occurrence of multiple independent disturbances. In the second step, the probabilistic model of the selected disturbance is used to generate a sample or event. This sample or event, when read into the simulation tool, will be interpreted as, e.g., a generator failure, and the generator power output will decrease to zero following a curve similar to the one shown in Fig. 1.

\section{A. Probability/Frequency Calculation of High Order Disturbances}

Implementation of the first step requires the development of a list containing all credible single disturbances and their combinations, and the associated frequencies or probabilities. This disturbance list includes the generator (a conventional generator or a solar generator) trips, the fast solar transients, and the combinations of these disturbances. Since the transient responses are of interest, the repair of these outages does not need to be considered and the mitigation comes only from the droop control of healthy generators and ESSs, if deployed.

The number of combinations increases exponentially with the number of single disturbances. The disturbance list has to be manageable by either discarding the high order combinations and/or truncating the combinations based on probabilities of disturbances using a threshold value. In this study, only second order disturbances, i.e., combinations of two disturbances, are considered because of mandated satisfaction of the $(N-1)$-criterion in planning. Higher order disturbances can be modeled similarly if needed.

Typically, a component may have multiple failure modes with different effects or fail from one mode to another. For example, a generator may experience a de-rated generation over an extended time period before a complete loss of generation. To facilitate discussions, an assumption made here is that a component will fail only once. This assumption is believed to hold for this study because the transients will be completed within seconds. It would not add much value to this study to assume that a component fails more than once.

Modeling independent component failures is considered first. Based on the above assumption, a Markov model shown in Fig. 3 is used to calculate failure probabilities for each component [14]. For a system consisting of $M$ components and each component has $N_{i}, i \in[1, M]$, failure modes (states), which can be represented as $C_{(i, j)}, \forall i \in[1, M]$, $j \in\left[0, N_{i}\right] . \lambda_{(i, j)}$ indicates the transition rate from the normal state of Component $i$ to its $j^{\text {th }}$ failure mode. We note that $C_{(i, 0)}, i \in[1, M]$ indicates the component's normal state, i.e., there is no failure with Component $i$. 


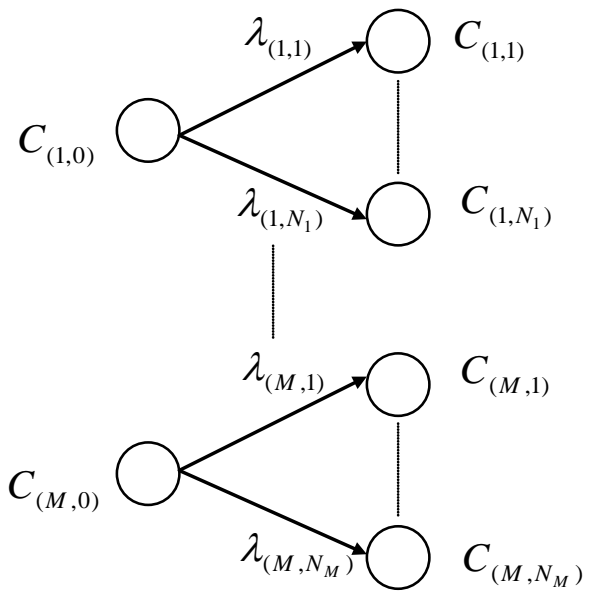

Fig. 3. Individual Markov models for $M$ independent components.

The probability of any state of a component can be calculated as:

$$
\begin{aligned}
& P\left(C_{(i, 0)}, t\right)=e^{-\lambda_{i} t} \\
& P\left(C_{(i, j)}, t\right)=\frac{\lambda_{(i, j)}}{\lambda_{i}}\left(1-e^{-\lambda_{i} t}\right)
\end{aligned}
$$

where $\lambda_{i}=\sum_{u=1}^{N_{i}} \lambda_{(i, u)} \quad$ for $\quad i \in[1, M], j \in\left[0, N_{i}\right]$, $0 \leq t<\infty$. A system state is represented by the states of all components and can be written as $C_{\left(i_{1}, j_{1}\right)} \quad C_{\left(i_{2}, j_{2}\right)} \quad \cdots$ $C_{\left(i_{M}, j_{M}\right)}, i_{k} \in[1, M], j_{k} \in\left[0, N_{i_{k}}\right]$ with $k=1,2, \cdots, M$. According to the assumption of failure independency, the probability of the system in a particular state is given as

$$
\begin{gathered}
P\left(C_{\left(i_{1}, 0\right)} \cdots C_{\left(i_{k}, j_{k}\right)} \cdots C_{\left(i_{l}, j_{l}\right)} \cdots C_{\left(i_{M}, 0\right)}, t\right) \\
=P\left(C_{\left(i_{k}, j_{k}\right)}, t\right) \cdots P\left(C_{\left(i_{l}, j_{l}\right)}, t\right) \prod_{\substack{u=1 \\
u \neq i_{k} \\
\cdots \\
u \neq i_{l}}}^{M} P\left(C_{(u, 0)}, t\right)
\end{gathered}
$$

A CCF may involve a number of different components and also have different failure modes. It can be modeled separately from the individual components by treating the CCF of a particular number of components as a new component, as shown in [11]. Similar to individual components, a Markov model is used to describe the CCF for this new component with the failure rates obtained from the CCF data, e.g., a $\beta$ factor CCF model [12] using the individual component failure rate. Therefore, equations (1) and (2) are still applicable to all of the meaningful states, i.e., a system state should not contain both the individual failure of a component and the CCF of the same component. A dependent failure, although not considered in this study, can be modeled similarly.

Theoretically, all of possible combinations of component failure modes need to be considered in the evaluation, i.e., a state enumeration approach [11]. In practice, it is difficult to considered high order failures for a relatively large system and unnecessary because the higher order failures are generally less likely to occur.

\section{B. Data Collection and Analyses for Generator Failures and Fast Solar Transients}

In general, there is a lack of the study of component failure parameters from different sources regardless of the existence of some databases (e.g., see [15, 16]). For this proof-of theconcept study, the values from these example sources are simply adopted without detailed analysis. The mean value of the frequency of a single generator trip is estimated to be 4 times per year in [15], i.e., a failure rate of 4.6E-4/hour. Due to a lack of data, a uniform distribution is assumed for the generator trip time between 0 and 2 seconds.

A solar plant may have two failure modes, i.e., a loss of generation and a fast solar transient. The solar plant trip may be due to many different causes and the failure rate can be estimated by investigating the frequencies of these causes. Because of the distributed nature of the inverters and arrays, the likelihood of simultaneous losses of all generation is anticipated to be very low. It is postulated that the failures of step-up transformers and the associated cables are dominant causes and the failure rate of a solar plant is estimated to be less than 6.0E-6 per hour using the data in [17].

The solar transient frequency was obtained in this study using the high-resolution (1-sec) solar resource data from a solar farm collected in a one-year period of 2011. A fast transient is defined as a transient that starts with the solar irradiance above $500 \mathrm{~W} / \mathrm{m}^{2}$ and the percentage of deviation from the initial value is above $50 \%$ within 15 seconds. The values selected in this definition are relatively arbitrary and can be refined. By applying this criterion, a total number of 2,283 transients were identified from the actual measurement data within one year. Note, this just means a portion of the plant experiences the fast transients.

To check the correlation of the random variables representing irradiance values, percentage of deviations, and transient times, scatter plots of these parameters in pairs are shown in Fig. 4. Fig. 4 indicates that three parameters appear to be weakly correlated since the circles spread over the plot. The transient time is not continuous because of resolution, i.e., 1-second time interval of the solar resource data.

Correlation coefficient is commonly used to quantify the relationship between random variables in statistics [18]. Correlation coefficient is between -1 (negatively correlated perfectly) and +1 (positively correlated perfectly) and the smaller its absolute value is, the weaker the correlation between two random variables becomes. The correlation coefficients between three sets of data are calculated [18] and shown in Table 1. 

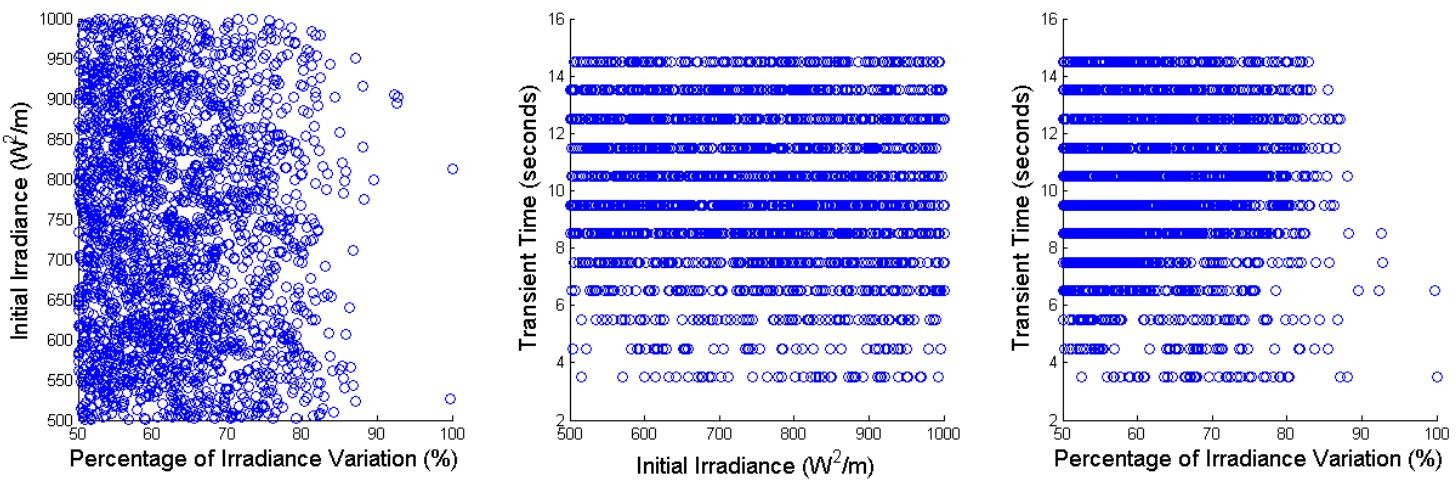

Fig. 4. Scatter-plots for solar transient parameters.

Table 1: Correlation Coefficients between Random Variables for Solar Transients

\begin{tabular}{|l|l|l|l|}
\hline & $\begin{array}{l}\text { Initial } \\
\text { Irradiance }\end{array}$ & $\begin{array}{l}\text { Percentage of } \\
\text { Irradiance } \\
\text { Variation }\end{array}$ & $\begin{array}{l}\text { Transient } \\
\text { Times }\end{array}$ \\
\hline Initial Irradiance & 1.0 & -0.049 & -0.067 \\
\hline $\begin{array}{l}\text { Percentage of Irradiance } \\
\text { Variation }\end{array}$ & -0.049 & 1.0 & 0.037 \\
\hline Transient Times & -0.067 & 0.037 & 1.0 \\
\hline
\end{tabular}

The results in Table 1 show that the initial irradiance values and the deviation percentage are only very weakly correlated while both initial irradiance and percentage of irradiance deviation are almost completely decoupled from the transient times. Based on this observation and also, for simplicity, three independent random variables $r . v_{1}, r . v_{2}$, and $r . v_{4}$ in Section II.A are used to describe the solar transients.

The initial irradiance values, the percentage of irradiance changes during the transients, and the transient times were sorted separately and are also plotted in Fig. 5. The histograms are further binned using intervals of $10 \mathrm{~W} / \mathrm{m}^{2}$ for initial irradiances, $1 \%$ for percentage of irradiance variations, and 1 second for the transient time, as shown in Fig. 6, which can be used to develop the discrete distributions for these random variables. Since the purpose of this study is to demonstrate the approach, we choose to describe the random variables all with uniform distributions ${ }^{2}$, i.e., of ranges $[500,1000]$ for initial irradiances $\left(\mathrm{W} / \mathrm{m}^{2}\right),[0.5,0.9]$ for percentage of irradiance variations (i.e., $50 \%$ to $90 \%$ ), and [3.5, 14.5] for solar transient times (seconds), respectively.

Next step is to capture the power variations of the entire plant using a modeling approach. It is assumed that (a) the solar plant consists of a number of arrays (e.g., eight arrays in this study) and all of them are connected to the selected bus;

\footnotetext{
${ }^{2}$ Data plotted in Fig. 6 can be approximated by some types of analytical distributions. For example, the frequencies versus cloud transient times may fit a normal distribution may fit, e.g., with $\mu=10.2$ (the sample mean) and $\sigma=2.7$ (the sample standard deviation). If a normal distribution is adopted, the random variates or samples for the Monte Carlo simulation can be generated using, e.g., an inverse transform method [9]. Note that the normal distribution has to be truncated.
}

(b) each array is equipped with a power conversion system (PCS) interfacing with the grid; and (c) the irradiance level at all panels of a particular array is the same at a given time instant [12]. To avoid considering high order sequences for the solar arrays, the occurrence rate of the fast transients for the entire solar plant is roughly estimated below.
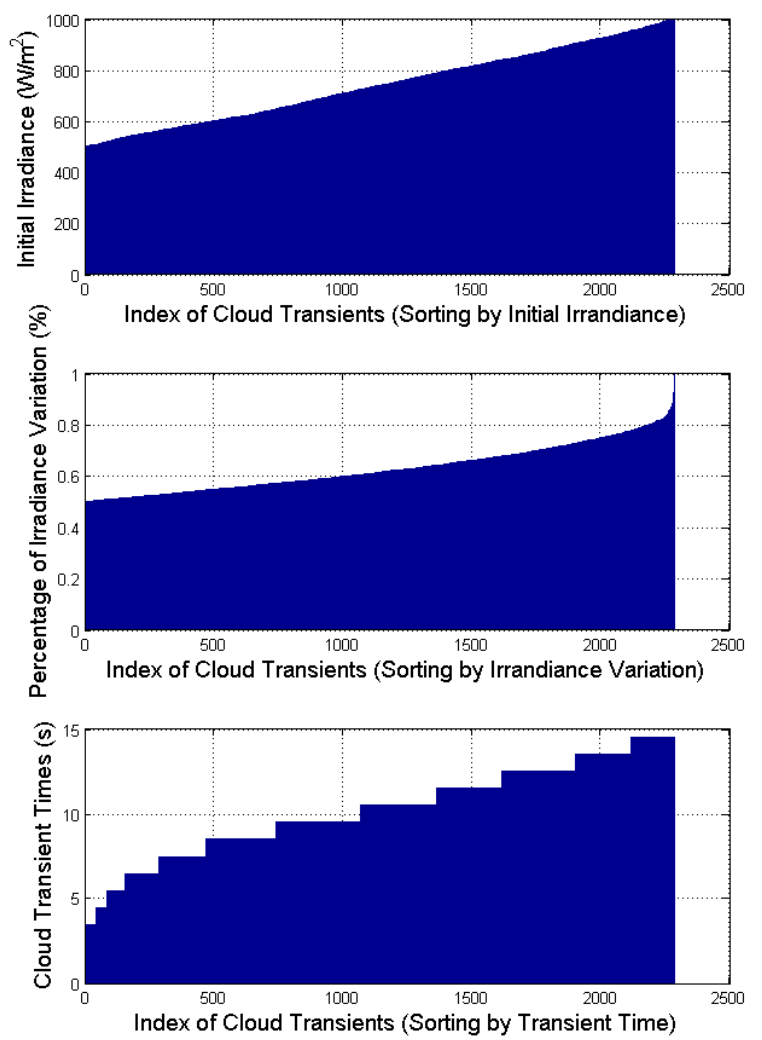

Fig. 5. Histograms of sorted initial irradiance, percentage of irradiance deviation, and transient times from collected data.

The occurrence rate of a fast solar transient is about $2,283 /(8,760 / 2)=0.52$ per hour. Using equation (1), the hourly probability of a fast solar transient $P_{S T}$ is approximately 
$P_{S T}(t)=1-e^{-0.52 \times 1}=0.41$ and the probability of simultaneous occurrence of fast transients at all eight arrays $P_{S S T}(t)$ is given by $P_{S S T}(t)=0.41^{8}=7.4 \mathrm{E}-4$ per hour. The corresponding frequency, i.e. the expected number in a time period $(0, T)$ can be evaluated as
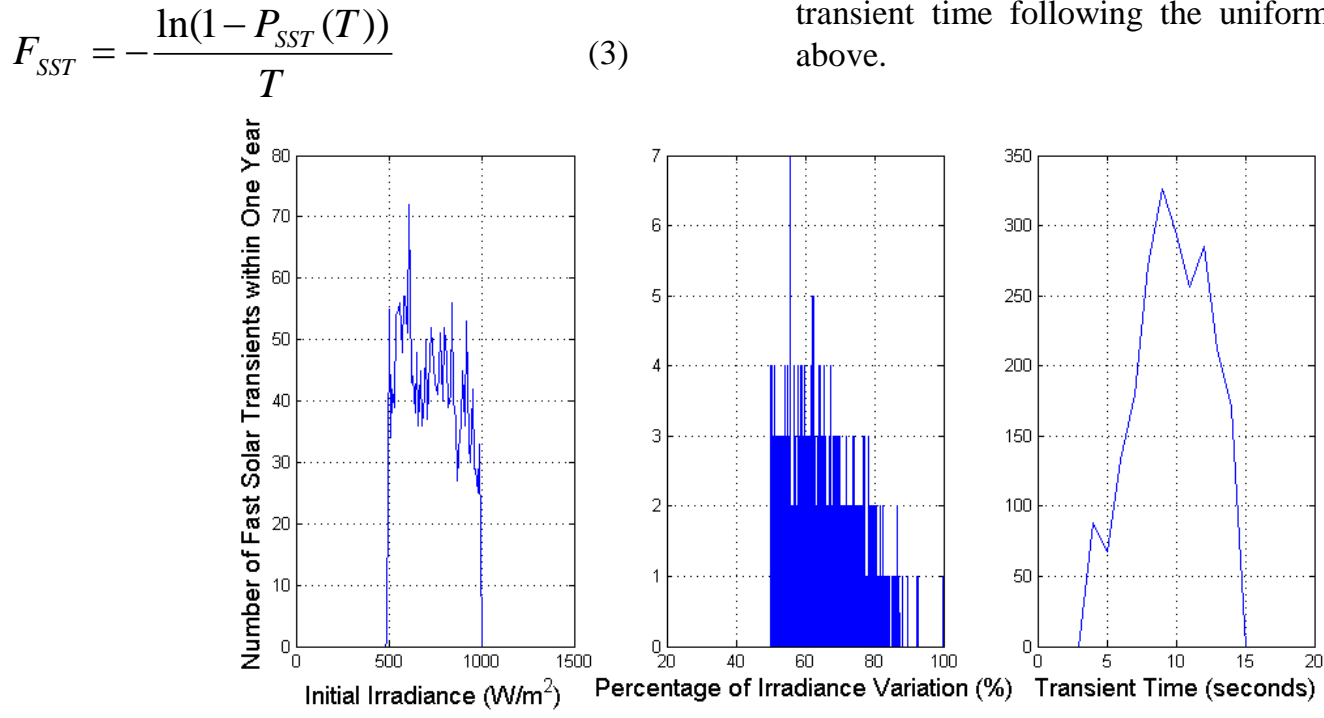

Fig. 6. Number of solar transients versus irradiance deviation from collected data. assuming that the solar transient occurrence follows a Poisson process with a constant rate [14]. The fast transient frequency of 7.4E-4 per hour or 3.2 times per year is the frequency of the fast transients experienced by the entire solar plant and when this occurs, there will be a transient at each array with the initial irradiance, percentage of irradiance deviation, and the transient time following the uniform distributions discussed above.
Note, the transient at a solar plant is considered a single or first order disturbance ${ }^{3}$. A summary of failure parameters used in this study for different failure modes of conventional generators and solar plants is shown in Table 1.

Table 1: Failure parameters of generators and solar plants

\begin{tabular}{|l|c|c|c|c|c|}
\hline Components & Generator & \multicolumn{2}{|c|}{ Solar Plant } & Generator $\begin{array}{c}\text { Solar } \\
\text { Plant }\end{array}$ \\
\hline $\begin{array}{l}\text { Failure } \\
\text { modes }\end{array}$ & Trip & Transient & Trip & CCF $\beta$-factor \\
\hline Failure & $\begin{array}{c}4.6 \mathrm{E}- \\
\text { 4/hour }\end{array}$ & $\begin{array}{c}7.4 \mathrm{E}- \\
\text { 4/hour }\end{array}$ & $\begin{array}{c}6.0 \mathrm{E}- \\
6 / \mathrm{hour}\end{array}$ & 0.1 \\
parameters & {$[15]$} & $\begin{array}{c}\text { Collected } \\
\text { data }\end{array}$ & {$[17]$} & {$[11]$} \\
\hline Data sources & & & \\
\hline
\end{tabular}

Since the second-order disturbances are not mutually exclusive or independent, their total probability can only be calculated exactly using the inclusion-exclusion principle [14], which may be difficult because the large amount computational efforts are required when the number of disturbances is large. If the failure probabilities of disturbances are small, the minimum cutset upper bound or even the rare event approximation can be applied to estimate the union probability of all disturbances [19]. Using the minimum cutset upper bound approximation, the total probability of second-order disturbances is around 7.8E-5 per hour, which indicates the likelihood of having a second order

\footnotetext{
${ }^{3}$ Such an aggregated model accounting for the irradiance variations at individual arrays is fairly crude. If the high-resolution data for the solar power output is available at each array, the statistics of the plant output can be easily obtained by convoluting the distributions at individual arrays.
}

disturbance is high and more attention should be paid to them.

\section{SAMPLE GENERATION AND PROCEDURES FOR BESS CAPACITY DETERMINATION}

\section{A. Sample Generation of High Order Disturbances for a Monte Carlo Simulation}

Using equations (1) and (2) the probabilities of all disturbances can be calculated and their frequencies can be obtained using equation (3). Once the total sample number for the Monte Carlo simulation is determined, the number will be distributed to individual disturbances proportionally to their occurrence frequencies.

A solar transient occurring at a plant means that all solar arrays experience transients of a same initial irradiance but different percentages of irradiance deviation and transient times, and uniform distributions discussed in previous section will be used to reconstruct the irradiance profiles. The irradiance profile during a cloud transient is generated using a three-segment high-order polynomial, i.e., a 4-3-4 polynomial that is often used for a trajectory planning in a joint space of a robot [20]. The random numbers for initial irradiance levels, percentages of irradiance decrease, and transient times to the lowest irradiance level are used to derive parameters for the polynomial to fit the irradiance profile. For a generator trip, a random time between 0 and 2 seconds, i.e., unif $(0,2)$, will be selected to determine the incremental internal impedance of the generator. In summary, the samples for the Monte Carlo 
simulation are generated offline as follows:

1) Develop a list of all second-order disturbances; Calculate the probability of each disturbance's occurrence using equations (1) and (2); Disturbances with a probability larger than the selected truncation threshold are included ( $10^{-11}$ in this study);

2) Convert the probability to frequency $F_{i}$ using equation (3) and calculate normalized frequencies $\bar{F}_{i}$ such that $\sum_{i \in \Omega_{S}} \bar{F}_{i}$ $=1.0$, where $\Omega_{S}$ is the set of all second-order disturbances;

3) Determine the total number of samples;

4) Generate a random number of uniform distribution $R=$ unif $(0,1)$, select the second order disturbance, i.e., if $\bar{F}_{i}<R \leq \bar{F}_{i+1}$, then the $(i+1)^{\text {th }}$ disturbance is selected;

5) If the selected second-order disturbance contains

a. a generator failure (or a CCF of two generators), the time it takes the generator (or the two generators) to trip is of a uniform distribution unif $(0,2)$;

b. a solar transient, generate a single initial irradiance of unif $(500,1000)$ for all arrays of the plant but different percentages of irradiance variations of unif $(0.5,0.9)$, and transient times of unif $(3.5,14.5)$;

c. a solar plant failure (or a CCF of solar plants), generate an initial irradiance of unif $(500,1000)$, a $100 \%$ of irradiance decrease (i.e., a zero solar power output) with a transient time of unif $(0,2)$ for all arrays of the plant(s).

6) For each selected disturbance or sample, generate the generation ramp-down curve and/or irradiance profile using the probabilistic models in Section II; until all samples are generated.

Note that Step 4) guarantees that the sample numbers for individual disturbances are proportional to normalized frequencies. A non-sequential Monte Carlo simulation is used to ensure that each generated sample is a second-order disturbance since this study focuses on the CCF and simultaneous occurrences of two independent disturbances and repairs are irrelevant in the transient responses.

An online sample generation simulation may also be implemented using a convergence criterion or stopping rule [13], e.g., the coefficient of variance. In this study, an offline scheme is adopted to facilitate the implementation and the sample number is considered relatively large because the random variables are not correlated and there is no issue of the state-of-knowledge-correlation (SOKC) [21]. Confidence intervals are analyzed for simulation outcome assurance below for a given sample size.

\section{B. A Monte Carlo Simulation for BESS Capacity} Determination

Since, in many utilities' practices, under-frequency load shedding relays are initiated when the frequency is less than a certain value, e.g., a frequency nadir of $59.5 \mathrm{~Hz}$ or frequency deviation $\Delta f=0.5 \mathrm{~Hz}$, this value can be selected as the threshold at which the BESSs are needed. The procedures for performing the simulation are the follows:

1) Starting with zero BESS capacity and all samples are marked for evaluation;

2) For the given BESS capacity, simulate the frequency responses of the system using the generation ramp-down curves and/or solar irradiance profiles generated in Section IV.A for each sample scenario that needs evaluation;

3) For a sample scenario, if the largest frequency deviation is smaller than $\Delta f$, then it does not need to be evaluated and move to next sample scenario; otherwise, the scenario is marked for re-evaluation; Do this for all scenarios and record the BESS capacity and number of scenarios that need re-evaluation;.

4) For scenarios that need re-evaluation, increase the ESSs capacity in a step-wise manner;

5) Repeat from Step 2) until no sample causes frequency nadir lower than the selected threshold or the desired number of samples with frequency deviation less than $\Delta f$ is achieved.

\section{Analyses of Monte Carlo Simulation Outcomes}

For each total capacity of the BESSs, a point estimate of the probability of sufficient BESS capacity (denoted by $P_{S B C}$ ) can be easily calculated from the number of scenarios of frequency deviation smaller than $0.5 \mathrm{~Hz}$ (denoted by $n_{s f d}$ ) and the total number of samples $n$, i.e., $P_{S B C}=\frac{n_{s f d}}{n}$. This point estimate will not be exactly the same as the true probability. Instead, a confidence interval is often used with a probabilistic statement about the assurance that the parameter being estimated lies in the interval, i.e., confidence level (denoted by $c$ ) [22]. If a typical confidence level value of $95 \%$ is used, then we are $95 \%$ confident that the true value of $P_{S B C}$ is, approximately within the interval of

$$
P_{S B C} \pm 1.96 \sqrt{\frac{P_{S B C}\left(1-P_{S B C}\right)}{n}}
$$

for $n_{s f d} \geq 5$ and $\left(n-n_{s f d}\right) \geq 5$. If $n_{s f d}<5$ or $\left(n-n_{s f d}\right)$ $<5$, the upper and lower bounds are approximated by

$$
\frac{\left(n_{s f d}+1\right) \times F I N V_{0.975}\left(2\left(n_{s f d}+1\right), 2\left(n-n_{s f d}\right)\right)}{\left(n-n_{s f d}\right)+\left(n_{s f d}+1\right) \times F I N V_{0.975}\left(2\left(n_{s f d}+1\right), 2\left(n-n_{s f d}\right)\right)}
$$

and 


$\frac{n_{s f d}}{n_{s f d}+\left(n-n_{s f d}+1\right) \times F I N V_{0.975}\left(2\left(n-n_{s f d}+1\right), 2 n_{s f d}\right)}$,

respectively, where $F I N V_{q}$ is the $q^{\text {th }}$-quartile of the $F$ distribution [18]. For $n_{s f d}=n$, a upper bound of 1.0 cannot be ruled out and the lower confidence bound $e^{\frac{\ln (1-c)}{n}}$ with a confidence level $c$ [18].

\section{RESUlts OF A CASE STUdY}

To simulate the frequency responses, dynamic models for solar plants, BESSs, and interfaces and their controls were developed. The details including a solar and lead-acid battery cells model being used to represent the solar plant and BESS can be found in [12] and are not discussed here. All of the models including the procedures for BESS capacity determination have been implemented and integrated into a Matlab-based power system simulation software EPTOOL that was developed based on the Power System Toolbox [23].

A 16-machine 68-bus power system [24] with a total generation about $18,000 \mathrm{MW}$ is used as the example system (see Fig. 7) in this study. All of the 16 generators (connected to buses $53-68$ ) are equipped with turbine governor controls.

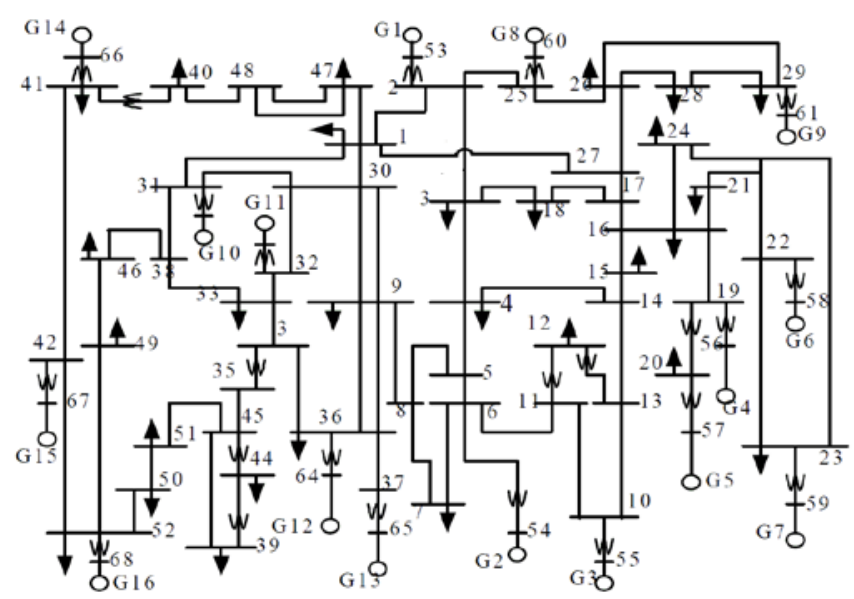

Fig. 7. The diagram of a 16-machine 68-bus example system.

Three identical solar PV plants contribute to about 35\% solar penetration level of the system at three buses that are the receiving ends of three transmission lines with the highest losses [12]. The penetration level is defined here as the ratio of solar generation under the standard condition (i.e., 1,000 $\mathrm{W} / \mathrm{m}^{2}$ and $25 \mathrm{C}^{\circ}$ ) to the total system generation. Three identical BESSs with droop frequency control can be deployed and connected to three different buses in the system, if needed. This may not necessarily lead to an optimal placement of the BESSs. However, the benefit associated with this deployment is also obvious because, in addition to provision of a frequency regulation capability, the BESSs are also able to cope with the intermittency of the solar plants. Also, since the three PV plants are identical, we assume the same capacity for each BESS. Each of three solar plants is divided into 8 arrays (i.e., arrays $1-8,9-16$, and 17 - 24 for solar plants 1, 2, and 3 , respectively). To achieve a 35\% penetration level, each of the 24 arrays is assumed to consist of 20 by 60,000 BP 3225 modules in serial and parallel connections.

When integrating the solar plants into the grid, the total generation is maintained by removing generators 1 and 2 (connected to buses 53 and 54) from the original system and cutting the generation, as needed, at the rest of the conventional generators proportionally. Therefore, the grid inertia decreases as solar penetration level increases.

A total number of 1,000 samples containing second-order disturbances only were generated for the Monte Carlo simulation. Among them, 156 samples contain a solar transient of one or more solar plants while the rest of the samples consist of either common cause failures or simultaneous (independent) failures of two generators, which indicates that the major contribution to the disturbances is from the generator outages in this example study.

A fast solar transient example is shown using one of the samples to illustrate the impact a generator outage under different scenarios. In the example, the generator outage is the loss of generator at bus 58. The scenarios are: (1) without solar generation, i.e., the original system inertia is maintained and solar generation is zero; (2) with solar generation, i.e., the reduced inertia due to the removal of machines at buses 53 and 54 and a solar transient at solar plant 3 with the irradiance profiles of 8 arrays of this plant shown in Fig. 8; and (3) the same as scenario (2) but with three ESSs connected to the three PV plants. Note, the effects of the transients will be reflected in the variation of the plant output that is the sum of generation at eight arrays. The other two solar plants do not experience any transient and continue to generate constant power outputs.

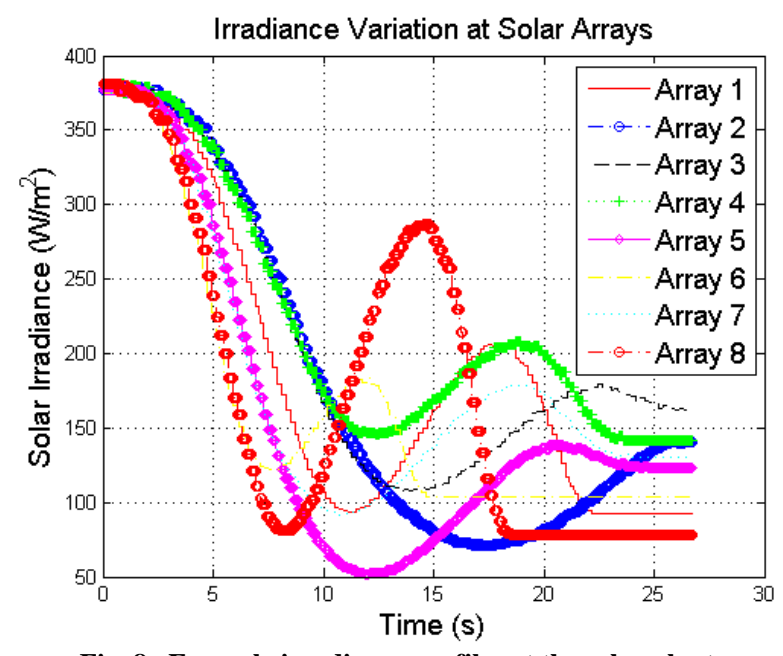

Fig. 8. Example irradiance profiles at the solar plants.

The frequency deviations of generator at bus 55 are shown in Fig. 9 for the above three scenarios (dashed line, dashed and 
dotted line, and the solid line, respectively, in Fig. 9). The loss of generator at bus 58 occurs at about 12.1 seconds in all scenarios. Scenario 1 (dashed line in Fig. 9) actually represents a single failure and the frequency deviation is slightly larger than $0.1 \mathrm{~Hz}$, which is of no concern to the operation. This can also be used to justify that all single disturbances are not included in the study. In scenarios 2 and 3 , the solar generation is included and the solar transient starts from time zero. Fig. 9 shows that, with the reduced inertia the solar transient impact alone (i.e., before the generator trip in Fig. 9) on the frequency is very small. This is because of the small changes in the irradiance profile shown in Fig. 8. However, the reduced inertia is the major concern in this case since the frequency deviation (the dashed dotted line) is larger than $0.7 \mathrm{~Hz}$. With the ESSs, the deviation can be brought back to around $0.3 \mathrm{~Hz}$ (the solid line). Larger solar transients are probably still possible, and, if coupled with generator disturbances, will pose much greater threat to the grid stability.

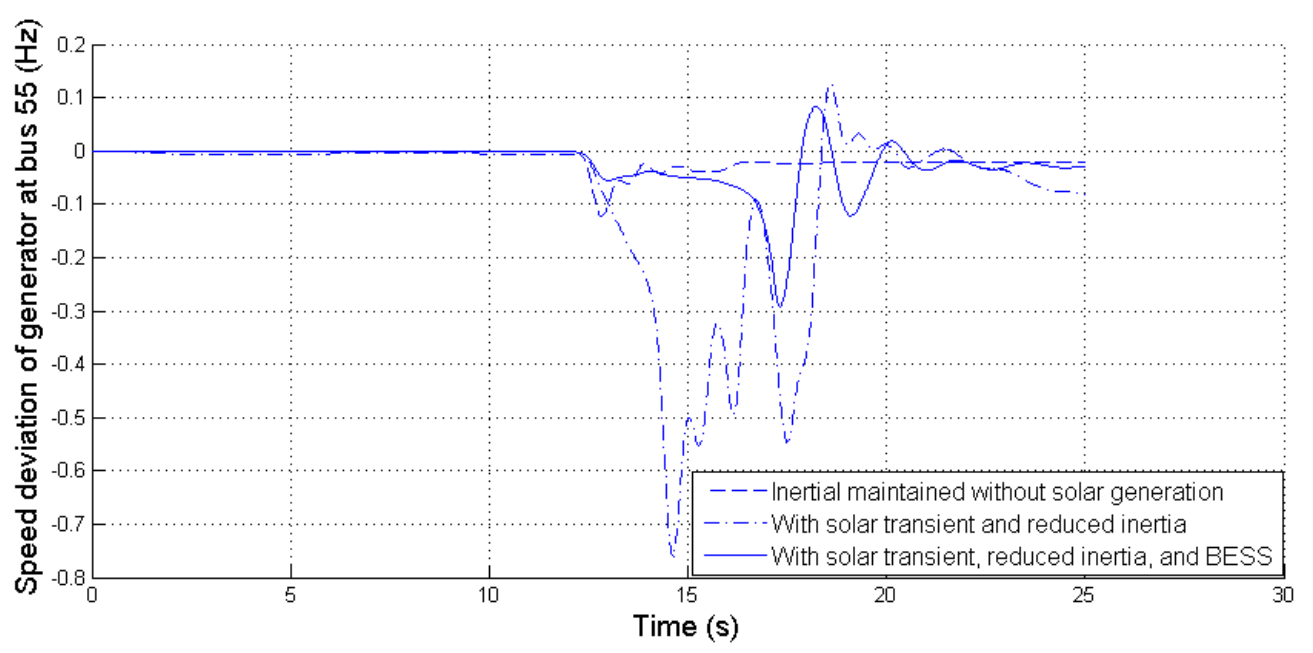

Fig. 9. Frequency deviations for various scenarios under a loss of generator.

The simulation of the generated samples was performed using the procedures defined in Section IV.B. The summary analyses of simulation outcomes are shown in Table 2. Note, an increase of BESS capacities is achieved by increasing the number of BESS cells, and the energy capacities are calculated simply as the summation of capacities of all battery cells. Also, the confidence intervals of the probabilities satisfying the frequency deviation threshold $P_{S B C}$ with a 95\% confidence level are calculated and shown in Table 2. The probabilities of meeting the threshold requirement can be calculated easily and shown in Fig. 10 together with the confidence intervals.

Table 2: Summary of Simulation Results (Total 1,000 Samples).

\begin{tabular}{|l|c|c|c|c|c|}
\hline Number of Scenarios & 946 & 977 & 980 & 981 & 983 \\
\hline $\begin{array}{l}\text { ESS Energy Capacities } \\
\text { (GWh) }\end{array}$ & 0 & 0.053 & 0.11 & 0.53 & 3.2 \\
\hline Confidence Interval of & {$[0.932$,} & {$[0.968$,} & {$[0.971$,} & {$[0.973$,} & {$[0.975$,} \\
95\% Confidence Level & $0.960]$ & $0.986]$ & $0.989]$ & $0.990]$ & $0.991]$ \\
\hline Number of Scenarios & 984 & 995 & 996 & 999 & 1,000 \\
\hline ESS Energy Capacities & 4.3 & 6.4 & 8.54 & 10.67 & 16.0 \\
(GWh) & & & & & \\
\hline Confidence Interval of & {$[0.976$,} & {$[0.990$,} & {$[0.990$,} & {$[0.994$,} & {$[0.997$,} \\
95\% Confidence Level & $0.992]$ & $0.999]$ & $0.999]$ & $1.0]$ & $1.0]$ \\
\hline
\end{tabular}

It is noted that the probability satisfying the requirement and the ESS capacity are proportional to each other but in a nonlinear manner. For example, a deployment of $0.053 \mathrm{GWh}$ ESSs will significantly increase the probability of satisfying the desired response, i.e., from 0.946 to 0.977 . In some other cases, the increase in the ESS capacity is not that effective, as can be seen from the required capacity increase from number of scenarios 999 to 1,000 in Table 2. While no general conclusion can or should be drawn from this result, it is clear that having extremely good performance requires a large amount of capacity and is very costly. One has to select an acceptable level of confidence on the satisfactory performance, which may be determined by considering both the cost of the ESSs (as well as power electronics) and the cost due to the potential losses associated with the unacceptable performance based on the studies using the results here.

These probabilities are conditional, i.e., conditioning on the occurrence of the second order disturbances. The implication of Fig. 10 is that, e.g., the probability of satisfying the frequency deviation requirement for the second order outages is about 0.984 for a total ESS capacity of $4.0 \mathrm{GWh}$. Since the type of batteries is already selected, only energy capacity is specified here. The power capacity is mainly determined by the capability of the power electronic devices, and in this study, it is assumed that the capacity of the power electronics is sufficiently large. It should also be pointed out that the required capacity is related to the performance of the controllers for the ESSs. The results here are therefore valid for the selected batteries and existing control designs in the example system.

The same set of samples was simulated using the original system (i.e., the same inertia of the 16-machines) and the same solar installation. The number of samples that cause 
unacceptable frequency deviation is reduced to 31 from 54, i.e., a probability of $96.9 \%$ satisfying the desired frequency response. If the solar installation is zero, which is equivalent to considering the second order disturbances of conventional generators only, only 10 of the 1,000 sample scenarios causes frequency issue. This indicates that the issue of degraded inertial response is mainly due to the inertia replacement with some contributions from the solar transients using the assumptions and data in this study.

The probabilistic results here can be used as the input to a risk-informed decision-making process, e.g., to decide and justify the need of BESSs, the desired capacity of BESSs and the associated cost, and alternative options (and cost) to enhance the inertial responses.

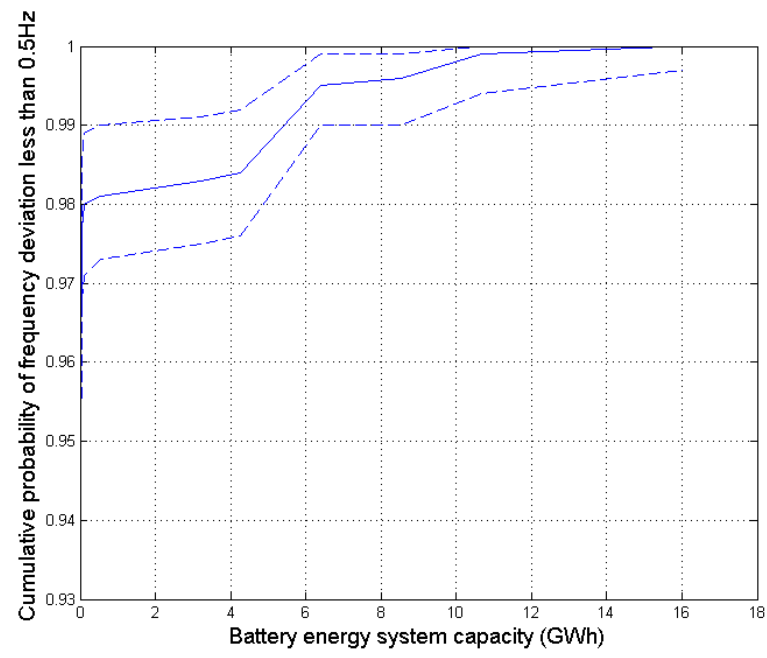

Fig. 10. Cumulative probabilities of satisfying frequency nadir requirement versus BESS capacities.

In the simulation, the initial state-of-charge (SOC) for ESSs is assumed to be the same, i.e., 0.7, considering that, in the transients, the BESSs may need to both deliver to and absorb energy from the grid. Once the SOC is less than 0.3, the BESSs will be disabled, as assumed in the simulation. Note, since automatic generation control (AGC) is not modeled, the frequency deviation as well as the demand signal for the BESSs will not become zero. In this study, the BESSs are not needed soon after the frequency starts to become stabilized.

Also, the initial operating conditions are the same in this study while, in practice, they always vary but may be described by distributions developed from the historic data. All these effects may be accounted for by including more randomness in the study. The energy capacities of three ESSs are the same and collocated with the three solar plants for the purpose of both smoothing out the solar plant and supporting the inertial responses as needed. More samples could be generated and evaluated easily since the entire procedure has been automated.

\section{CONCLUSIONS AND FUTURE WORK}

In this study, a probabilistic approach is proposed to determine the ESS capacities from a perspective of the system response, i.e., the inertial response. The unique features of this study are that the study (1) attempts to address the degrading inertial responses that are difficult to correct using means other than the responsive energy storage systems, which justifies the particular need for costly energy storage systems; (2) determines the needed capacity from the perspective of the overall system operation, i.e., the impacts of the increasing penetration level and the variability of solar generation other than just smoothing out the intermittent generation of individual solar plants; and (3) considers not only high order outages but also the associated likelihood of their occurrences, which serves as the basis to sizing the ESSs in this study.

Results from this study show that this approach can be used to size the ESSs that mitigate frequency impacts on the grid in cases of reduced grid inertia and large power mismatches introduced by undispatchable renewable sources. Such remedies cannot be done by the conventional generators, as shown in this study. The proposed approach (1) is ready to be generalized to capture the randomness of any disturbances relevant to studies of different purposes and can be easily extended to different applications, e.g., the acceptable level of solar penetration in a given system or the desired battery capacity (and near-minimum cost) to achieve an acceptable grid inertial response;; (2) permits a straightforward implementation of the proposed approach using the existing commercial software packages; (3) enables a risk-informed decision making process if a risk measure is defined appropriately.

Note that the approach does not guide the search in the optimal direction. Due to the iterative nature of the approach, if multiple options are available, for example, deploying BESSs and/or building new generators, many more scenarios consisting of combinations of different BESS capacities and different number of generators may have to be evaluated to find a satisfactory solution.

For the proposed approach, evaluation of transient simulation using a large number of samples is unavoidable, which entails an extensive computational effort. Using fast computers is an option; but more importantly, it should be noted that the major feature of the Monte Carlo simulation is that the samples, after being generated offline, can be evaluated independently on a number of computers. This actually indicates an almost linear scalability of the computation as long as a tool for automating such a process is developed. The number of samples is, therefore, not of a severe concern, if the samples are evaluated in a distributed manner. In this study, the 1,000 samples were actually distributed to three computers to evaluate the inertial responses using the same incremental ESS capacity. This, 
although a manual process, has significantly reduced the total computational time needed for completion of the entire study.

It should be stressed that numerical values in this study are only used for demonstration purposes. Additional work to refine this approach includes a systematic statistical analysis of solar cloud transients using more real data and the variations of the initial conditions including load levels. Distributions of irradiance variation accounting for both location and timing will be investigated to better represent the fast transients experienced by solar plants. An improved control scheme for the BESSs to provide virtual inertia is being investigated and expected to improve the control performance and reduce the need for the ESS capacity. Different probabilistic measures for the inertia response and cost-benefit studies will also be investigated.

The models and the approach are being implemented in a commercial software package to speed up the calculations. In addition, a meaningful comparison between the approach proposed in this study and other methods, for examples, the deterministic methods described in [3] can be performed to better validate the approach.

\section{REFERENCES}

[1] J. Eto et al, "Use of Frequency Response Metrics to Assess the Planning and Operating Requirements for Reliable Integration of Variable Renewable Generation,” LBNL-4142E, Dec. 2010.

[2] N. Miller et al, "Frequency Response Study," a report for California ISO (CAISO), Nov., 2011.

[3] Bagen and R. Billinton, "Incorporating Well-being Considerations in Generating Systems Using Energy Storage,” IEEE Tracstions on Power Systems, Vol. 20, No. 1, March, 2005.

[4] L. Grimsmo, M. Korpaas, and T. Gjengdal, "Probabilistic Sizing of Wind and Hydrogen Power Systems for Remote Areas," $15^{\text {th }}$ Power System Computation Conference (PSCC), August, 2005.

[5] C. Abbey and G. Joos, "A Stochastic Optimization Approach to Rating of Energy Storage Systems in Wind-Diesel Isolated Grids, " IEEE Transactions on Power Systems, Vol. 24, No. 1, February, 2009.

[6] P. Pinson et al, "Dynamic Sizing of Energy Storage for Hedging Wind Powre Forecast Uncertainty,” IEEE Power \& Energy Society General Meeting, July, 2009.

[7] S. H. Karaki et al, "Probabilistic Performance Assessment of Autonomous Solar-Wind Energy Conversion Systems," IEEE Transactions on Energy Conversion, Vol. 14, No. 3, 1999.

[8] X. Wang, M. Yue, and E. Muljadi, "Probabilistic Approach for Power Capacity Specification of Wind Energy Storage Systems," IEEE Transactions on Industry Applications, Vol. 50, No. 2, 2014.

[9] H. Bludszuweit and J. Dominguez-Navarro, "A Probabilistic Method for Energy Storage Sizing Based on Wind Power Forecast Uncertainty, ” IEEE Transactions on Power Systems, Vol. 26, No. 3, 2011.

[10] P. Mercier, R. Cherkaoui, and A. Oudalov, "Optimizing a Battery Energy Storage System for Frequency Control Application in an Isolated Power System, ” IEEE Transactions on Power Systems, Vol. 24, No. 3, August, 2009.

[11] W. Li, "Risk Assessment of Power Systems: Models, Methods, and Applications,” IEEE Press Series on Power Engineering, 2005.

[12] J.W. Hickman et al., "PRA Procedures Guide: A Guide to the Performance of Probabilistic Risk Assessments for Nuclear Power Plants,” available online at http://www.nrc.gov/reading-rm/doccollections/nuregs/contract/cr2300/vol1/cr2300v1-b.pdf.
[13] M. Yue and X. Y. Wang, "Assessment of Cloud Transient Impacts of Grid-integratedSolar and Battery Energy Systems,” the Proceedings of 39th IEEE Photovoltaic Specialists Conference, 2013.

[14] T. Chu et al, "Modeling a Digital Feedwater Control System Using Tranditional Probabilstic Risk Assessment Methods," NUREG/CR6997, June, 2008.

[15] WSCC Reliability Subcommittee, "Probabilistic Based Reliability Criteria, Phase I: Event Probability Development and Implementation Plan,” an online report, 1998.

[16] Generating Availability Data System (GADS), available at http://www.nerc.com/page.php?cid=4|43.

[17] IEEE Guide to the Collection and Presentation of Electrical, Electronic, Sensoing Component, and Mechanical Equipment Reliability Data for Nlcear Power Generating Stations, IEEE, Inc, 1984.

[18] D. Lurie, L. Abramson, and J. Vail, “Applied Statistics,” NUREG-1745, March, 2011.

[19] C. L. Atwood et al, "Handbook of Parameter Estimation for Probabilistic Risk Assessment,” NUREG/CR-6823, 2002.

[20] K. S. Fu et al, "Robotics: Control, Sensing, Vision, and Intelligence," McGraw-Hill Education, 1988.

[21] T. L. Chu and M. Yue, "Analytical Calculation of the Mean Value of a Top Event," International Conference on Probabilistic Safety Assessment and Management (PSAM9), 2008.

[22] L. Leemis and S. Park, "Discrete-Event Simulation: A First Course," Prentice Hall, January, 2006.

[23] Power System Toolbox Webpage, http://www.ecse.rpi.edu /pst/PST.html.

[24] R. Graham, Power System Oscillations: Norwell, MA: Kluwer, 2000. 